\title{
Universality of jammed frictional packing
}

\author{
Ye Yuan (1) \\ College of Engineering, Peking University, Beijing 100871, China \\ and School of Physics and Astronomy, Shanghai Jiao Tong University, Shanghai 200240, China \\ Yang Jiao (1) \\ Materials Science and Engineering, Arizona State University, Tempe, Arizona 85287, USA \\ and Department of Physics, Arizona State University, Tempe, Arizona 85287, USA \\ Yujie Wang (1) \\ School of Physics and Astronomy, Shanghai Jiao Tong University, Shanghai 200240, China \\ Shuixiang $\mathrm{Li}$ (1) $^{*}$ \\ College of Engineering, Peking University, Beijing 100871, China
}

(Received 22 March 2021; revised 26 May 2021; accepted 4 June 2021; published 23 July 2021)

\begin{abstract}
Understanding disordered particle packings is of great significance from both theoretical and engineering perspectives. Establishing a quantitative relationship between nonspherical particle shape and disordered packing properties is generally challenging, due to the complex geometry and topology. Here we resolve this issue by numerically investigating disordered jammed packings of various frictional congruent nonspherical particles, including superellipsoids and polyhedra, over a wide range of friction coefficients. We discover several universal packing characteristics across different particle shapes and frictions. In the infinite friction limit, the coordination numbers for all shapes approach the identical lower bound for jamming. The resulting "random loose packing" (RLP) state possesses minimal structural correlations, with the packing fraction as a simple monotonic decreasing function of the orientation-averaged excluded volume for different particle shapes. Packings with finite friction can then be understood via a perturbative approach based on RLP. The nature of RLP can be illuminated by the percolation transition of contacting particle network during the quasistatic densification process. Moreover, the large-scale density fluctuations for all jammed frictional packings are also strongly suppressed, broadening the previous claim of hyperuniformity for the frictionless ones.
\end{abstract}

DOI: 10.1103/PhysRevResearch.3.033084

\section{INTRODUCTION}

Disordered particle packings are excellent models for a wide spectrum of condensed matter systems including lowtemperature states of matter, granular materials, colloids, emulsions, and foams [1-4]. Early in the 1960s, Bernal and co-workers experimentally found that monodisperse spherical granules in a container would form a stationary random packing under small perturbation, i.e., the random close packing with a robust packing fraction (i.e., fraction of space covered by the particles) $\phi_{J} \approx 0.64$ [5]. Since then, this particular packing state has been constantly reproduced by different numerical and experimental protocols, showing intriguing facets as the fluid-solid jamming transition of frictionless spherical granular particles under athermal compression or quasistatic

\footnotetext{
*1sx@pku.edu.cn

Published by the American Physical Society under the terms of the Creative Commons Attribution 4.0 International license. Further distribution of this work must maintain attribution to the author(s) and the published article's title, journal citation, and DOI.
}

shearing [2], the inherent structures of hard-sphere glasses with the fastest cooling rate [3,6], etc. Beyond a specific physical system, random close packing was also conceptually redefined as the maximally random jammed (MRJ) state using a geometric approach [3], with the highlighted hallmark of hyperuniformity, i.e., static structure factor vanishes in the zero wave-number limit $[7,8]$.

Deviating from the perfect spherical shape, the disordered packing properties, especially $\phi_{J}$, would be significantly influenced by the nonspherical shape of constituent particles in three dimensions (3D) [3,4,9]. A typical example is that certain ellipsoidal shape can possess $\phi_{J} \approx 0.735$ drastically higher than that for a sphere, and even close to the $f c c / h c p$ packing fraction $\approx 0.74$ [10]. Nonetheless, predicting the macroscopic (disordered) packing properties (e.g., $\phi_{J}$ ) based on the nonspherical shape alone is challenging due to the complex geometry and topology of the resulting disordered packings $[3,4]$. To our knowledge, only a few studies attempted to address this issue [9,11]. An advance in our fundamental understanding of this problem would lead to the promising rational design of particle shapes for a broad spectrum of applications in vastly different systems. For instance, the dependency of mechanical strength [12] and plasticity 
[13] on particle shape for granular materials might be better understood treating the geometric packing information as an intermediary. Colloidal synthesis technique allows the systematic exploration of the shape-controlled glass transition $[14,15]$, where $\phi_{J}$ marks the critical state with fully arrested dynamics. Also, there exists an underlying interplay between self-assembly and disordered packing formation in the dense phases for nonspherical colloids [16,17].

A disordered packing generally implies that the packing structures are (globally) decorrelated to a certain extent, indicating the existence of a mean-field approach which is potentially useful to reconstruct the average local packing around a central particle [9]. Therefore, coordination number $z_{J}$ serves as the primary estimate for local packing compactness. This claim is supported by different experimental and numerical observations about the MRJ packings (for frictionless particles) that $\phi_{J}$ always increases beyond $\sim 0.64$ as the nonspherical particle shape initially deviates from a perfect sphere, accompanied by the growing $z_{J}$ beyond the isostatic $z_{J}=6$ for sphere [9-11,18]. But larger shape deviation from a sphere might lead to a decrease of $\phi_{J}$ since the excluded volume, i.e., another mean-field quantity besides $z_{J}$, starts to play a considerable role [19]. Phenomenologically, the tradeoff between $z_{J}$ and excluded volume explains the ubiquitous nonmonotonic relation between $\phi_{J}$ and a certain shape parameter (e.g., aspect ratio) for the MRJ packings of different nonspherical particles $[9,18]$, especially elongated particles such as prolates $[10,20]$. Similar observations were also made for noncircular shapes in two dimensions [21,22]. However, a systematic investigation of such effects is lacking, especially when coupled with different interparticle frictions [23-25].

Here we resolve these outstanding issues by numerically investigating 3D disordered jammed packings of a wide range of congruent (convex) nonspherical particles, including superellipsoidal [18,26,27] and polyhedral [28-31] models, with varying friction coefficient $\mu$. We extend the concept of random loose packing (RLP), originally proposed for spherical particles of $\mu \rightarrow \infty$ with $\phi_{J}(\mu \rightarrow \infty) \approx 0.56$ (denoted by $\left.\phi_{J}^{\infty}\right)$ [23,32-36], to nonspherical shapes. For these RLPs, the coordination number achieves the theoretical minimum 4 , in agreement with the frictional isostaticity requirement $[1,37]$. As a result, the associated packing fraction $\phi_{J}^{\infty}$ is simply a monotonically decreasing function of the dimensionless, orientation-averaged excluded volume $v_{\mathrm{ex}}$ [38] for different nonspherial particles. Additional universal behaviors in structural characteristics for finite $\mu$ are revealed based on the RLP state as a reference. We also find that $\phi_{J}^{\infty}$ can be approximated by the percolation transition of the contacting particle network [39-42] preceding jamming during the quasistatic densification process for all particle shapes and $\mu$. In addition, our numerical results suggest that the local density fluctuations are strongly suppressed in all jammed packings, reminiscent of the hyperuniformity previously reported for MRJ packings $[7,29,43]$.

\section{RESULTS}

\section{A. Generation of jammed frictional packings}

We consider 3D superellipsoidal [18,26,27,44] and polyhedral [29-31,45] particles which include a variety of (convex) nonspherical shapes. A superellipsoid with centroid located at the origin is defined by $|x|^{2 p}+\left|y / w_{1}\right|^{2 p}+\left|z / w_{2}\right|^{2 p}=1$, where $p$ is the deformation parameter, and $w_{1}(\leqslant 1)$ and $w_{2}(\geqslant 1)$ are the two aspect ratios. Here, we focus on superballs [46,47] $\left(w_{1}=w_{2}=1\right)$ and ellipsoids [10,48,49] $(p=$ 1 , including prolates with $w=w_{2}>w_{1}=1$, oblates with $w=w_{1}<w_{2}=1$, self-dual ellipsoids with $w_{1} w_{2}=1$, and other general ellipsoids with two different aspect ratios). For polyhedral particles, we investigate tetrahedron [29,50-53], cube [52], as well as different types of cuboids [54] with aspect ratios defined via the three side lengths similar to those of a superellipsoid. The size of a particle is quantified by $D_{v}$, which is the diameter of a sphere with equal volume to the particle.

As previously reported, numerically obtained jammed packings could be protocol dependent. For example, jamming transition in different numerical simulations can occur at quite different $\phi_{J}$ with varying initial configurations or compression rates [2,3]. Nonetheless, it has been established that a dilute gas configuration of spheres, athermally compressed (i.e., quenched without thermal equilibration), jams at the MRJ state ( $J$ point) as the starting point of the $J$ line for frictionless particles $[55,56]$, which can be achieved via both soft- and hard-particle models [7,57]. A similar conclusion was also established for nonspherical particles [18]. Robust numerical procedures have been developed to study jamming of frictional sphere, which can evolve from the MRJ to RLP states by continuously tuning the friction coefficient $\mu[23,35]$.

In this work, we implement 3D molecular dynamics (MD) for monodisperse spherical and nonspherical soft particles with static friction [58,59], to model quasistatic compression from a dilute gas configuration towards jamming [57]. For all the obtained packings, the distance to the exact jamming onset is $10^{-5} \lesssim \Delta \phi \lesssim 10^{-3}$ (i.e., interparticle overlaps are negligible) and thus, are representative of jammed states of hard particles with friction. See Appendix A for the simulation details. The friction coefficient $\mu$ is varied over almost 3 orders of magnitude in our simulations, from 0.01 to 50 . Consistent with previous studies, jammed packings with $\mu \geqslant 10$ are sufficiently representative and practically referred to as the RLP states. The jammed packings of frictionless superellipsoids are generated using the L-BFGS energy minimization method $[18,52,60,61]$. Note that the simulations of frictionless polyhedra show numerical instability close to jamming because of the model approximation. Thus these results are not included in this work. The typical particle numbers in the packings are $N=500-1000$ and larger systems $\left(N=10^{4}-10^{6}\right)$ are also investigated for assessing large-scale density fluctuations for certain shapes. The reported statistics are averaged over 5-10 independent configurations unless specified.

In Figs. 1(a)-1(h), we, respectively, show typical packing configurations of frictionless particles (the top panels) and particles with large friction (the bottom panels). All these packings are generally disordered. Nonetheless, we observe that the RLPs are much more random than the corresponding frictionless (MRJ) packings, with finite local stacking order and facet alignment observed in the later ones [Figs. 1(e) and 1(g)]. In Appendix B, two different orientational order metrics are quantified to support this argument. 

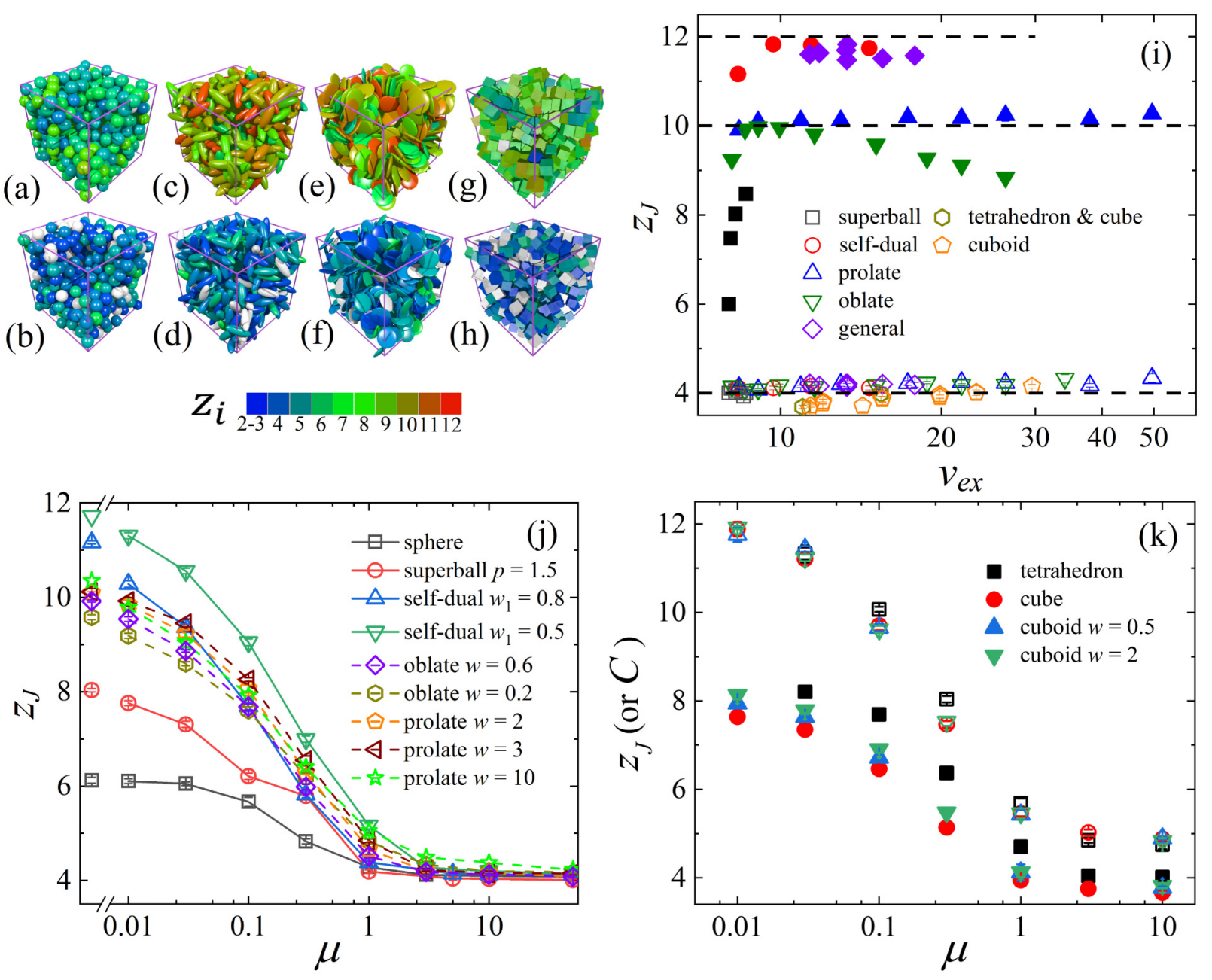

FIG. 1. Coordination statistics in jammed packings for different particles. Visualization of jammed packing configurations for (a) and (b) sphere, (c) and (d) prolate with $w=3$, (e) and (f) oblate with $w=0.2$, and (g) and (h) cube. $\mu=0$ for the top panels ( $\mu=0.01$ for cube as an exception) and $\mu=10$ for the bottom panels. Each individual particle is colored by its local contact number $z_{i}$ (see the color bar) and rattlers are in white. (i) Coordination number $z_{J}$ as a function of dimensionless excluded volume $v_{\mathrm{ex}}$ in frictionless (MRJ) packings (filled) and RLPs (open) for all the particles. Note that the filled symbols represent the MRJ states with the same particle shapes as the open ones. The diamond symbols denote general ellipsoids with two different aspect ratios except the self-dual ones with $w_{1} w_{2}=1$. Besides different superellipsoids, we also include several polyhedra (hexagons and pentagons). The dashed lines in (i) indicate $z_{\text {iso }}^{\infty}=4$ for RLPs, and $z_{\text {iso }}^{0}=10$ or 12 for packings of frictionless axisymmetric or nonaxisymmetric particles, respectively. (j) $z_{J}$ as a function of $\mu$ for eight selected superellipsoids. The leftmost data in (j) indicate the frictionless cases. (k) $z_{J}$ (filled) and constraint number $C$ (open) as a function of $\mu$ for four polyhedra.

\section{B. Universalities of packing characteristics}

The basic characterization for a packing structure is the coordination. In Figs. 1(a)-1(h), we color each particle $i$ according to its local contact number $z_{i}$ and find strong similarity in RLPs of distinct particle shapes. Note that RLPs typically contain a larger fraction of rattlers (i.e., particles not involved in the force-bearing backbone whose detection relies on a recursive algorithm, see Appendix A), which is about 10\%-15\% compared to their frictionless counterparts (less than 3\%-5\%). The rattlers are colored white in Figs. 1(a)-1(h) and they are excluded when measuring $z_{J}$. In Appendix $C$, we further show the relation between $z_{i}$ and local packing fraction, which is not universal. Thus, coordination alone cannot explain the complexity of packings.

On average, all the RLPs possess similar coordination numbers $z_{J}^{\infty} \approx 4$, regardless of the particle shapes as shown in Fig. 1(i), which validates the isostatic counting argument $z_{J}^{\infty}=z_{\text {iso }}^{\infty}=d+1$ as $\mu \rightarrow \infty[1,37]$. For frictionless superellipsoids, the coordination number $z_{J}^{0}$ of moderately aspherical particles could reach $z_{\text {iso }}^{0}=10$ (spheroids) or 12 (self-dual ellipsoids) [10], indicated by the dashed lines in Fig. 1(i). MRJ packings of nearly spherical particles are generally hypostatic with $6<z_{J}^{0}<12$, difficult to predict a priori $[18,48,62,63]$. Figure 1(j) shows that $z_{J}$ for eight selected superellipsoids will decrease from various $z_{J}^{0}$ and asymptotically approach the same $z_{\text {iso }}^{\infty}=4$ as $\mu$ increases.

For polyhedra, a certain contact can be classified into distinct types of contact topologies formed by vertexes, edges, and facets, which constrain different numbers of degrees of freedom, namely 3 for a facet-facet contact, 2 for an edgefacet contact, and 1 for the rest. Considering the summed constraint number $C$ (see Appendix A) for each particle on average, previous studies showed that $C \approx z_{\text {iso }}^{0}=12$ in 


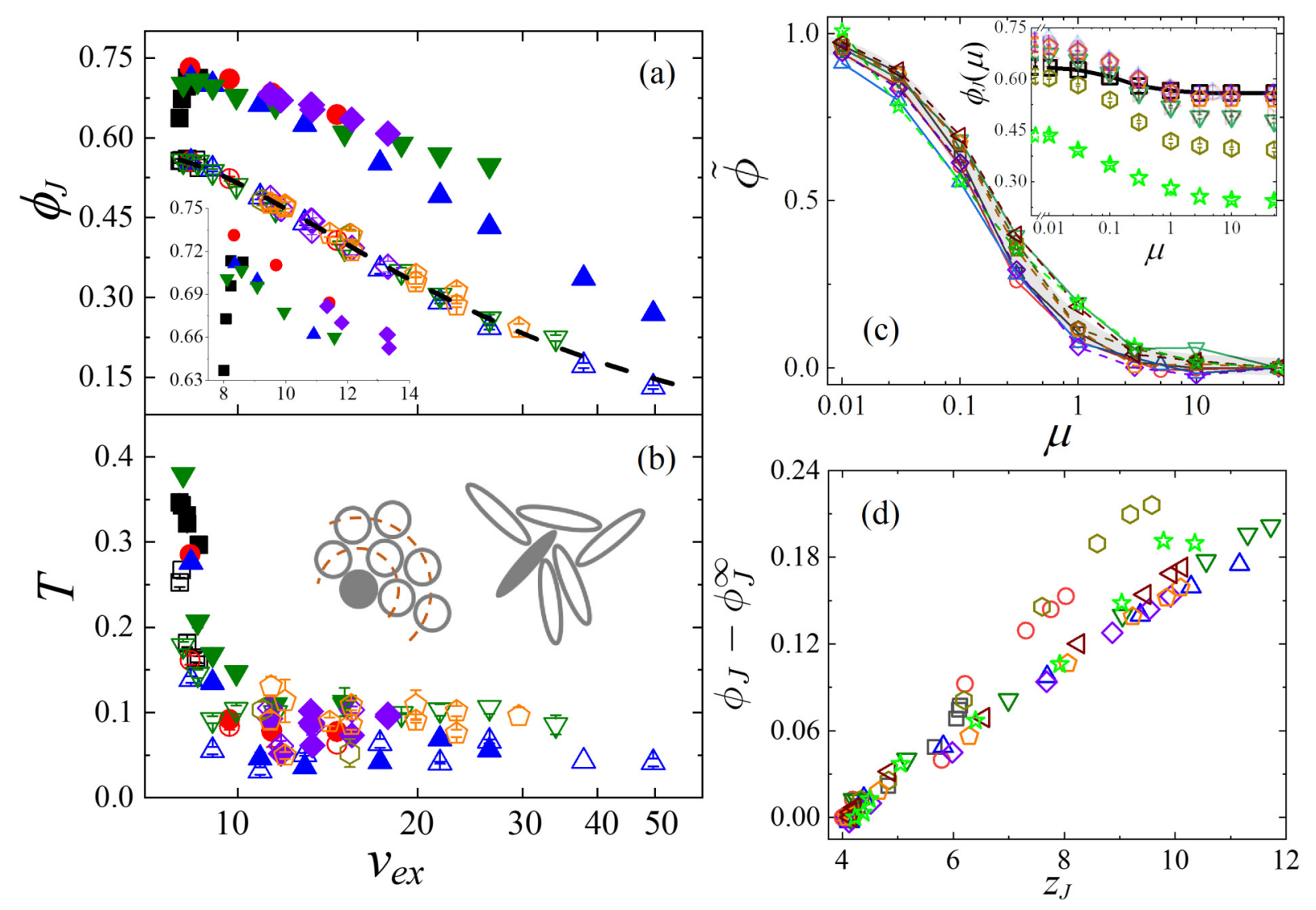

FIG. 2. Universalities of packing characteristics for different particles. (a) Jamming packing fraction $\phi_{J}$ and (b) translational order metric $T$ as a function of $v_{\mathrm{ex}}$ in frictionless packings (filled) and RLPs (open) for all the particles [in the same symbols as Fig. 1(i)]. The dashed curve in (a) indicates the fitting equation $\phi_{J}^{\infty}=c_{1} / v_{\mathrm{ex}}-c_{2} / v_{\mathrm{ex}}^{2}$. The inset in (a) shows the zoom-in of $8 \leqslant v_{\mathrm{ex}} \leqslant 14$. The 2D schematic in (b) compares local configurations in a disk packing and an ellipse packing. (c) Normalized packing fraction $\tilde{\phi}(\mu)$ and (d) excess packing fraction $\phi_{J}-\phi_{J}^{\infty}$ as a function of $z_{J}$ for eight selected particles [in the same symbols as Fig. 1(j)]. The thick curve in (c) indicates the fitting equation $\tilde{\phi}=\left[1+\left(\mu / \mu^{*}\right)^{\alpha}\right]^{-1}$. The inset of (c) shows the original results of $\phi_{J}(\mu)$.

jammed packings of frictionless polyhedra $[29,45,53,64]$. In our model, the constraint number for one contact between two polyhedra can be explicitly obtained without any geometric threshold. As shown in Fig. 1(k), both $z_{J}$ (filled) and $C$ (open) for four polyhedra show similar dependencies on $\mu$ as superellipsoids in Fig. 1(j). The fractions of different types of contact topologies as a function of $\mu$ for these polyhedra are given in Appendix D, Fig. 7(a). More importantly, in contrast with $z_{J}$, $C$ indeed approaches the isostatic value $z_{\text {iso }}^{0}=12$ as $\mu \rightarrow 0$ as a result of facet-facet and edge-facet contacts $[29,45,53,64]$. For large $\mu, C \gtrsim z_{J} \approx 4$ since the simple contacts dominate. These results strongly support the isostaticity of both RLP and MRJ for polyhedra.

For MRJ packings, previous studies have revealed complicated dependency of $\phi_{J}^{0}$ on particle shape $[9,18]$. Generally, $\phi_{J}^{0}$ will increase as the particle shape initially deviates from a perfect sphere, mainly due to the rapid increase of $z_{J}^{0}$ from $z_{J}^{0}=6$ [18]. For large shape deviations, $\phi_{J}^{0}$ becomes negatively correlated with asphericity and is dominated by effects of particle excluded volume [19]. Spheroids and spherocylinders with varying aspect ratios are good examples for this scenario $[10,20,48,65]$. In Fig. 2(a), $\phi_{J}^{0}$ (filled) displays such a nonmonotonic trend as $v_{\text {ex }}$ enlarged [18]. The inset as the zoom-in of $8 \leqslant v_{\text {ex }} \leqslant 14$ further shows the relatively large $\phi_{J}^{0}$ of self-dual ellipsoids owing to their $z_{J}^{0} \approx 12$. Note that $\phi_{J}$ for several polyhedra (including tetrahedron, cube, and cuboids) as $\mu \rightarrow 0$ generally agree with the previous numerical results [45,52-54] [see Appendix D, Fig. 7(b)]. Moreover, additional rotational degrees of freedom commonly require nongeneric and correlated particle arrangement for frictionless nonspherical packings [47], which might induce finite local order (Fig. 5). These coupled factors make it very difficult to quantitatively understand the relation between $\phi_{J}^{0}$ and particle shapes.

On the contrary, the isostatic $z_{J}^{\infty} \approx 4$ and minimally correlated local packing structures while maintaining jammed in RLPs inspire us to establish a simple quantitative relation between $\phi_{J}^{\infty}$ and the particle shape in a mean-field sense. Previous experimental and numerical studies have shown a robust correlation between the packing fraction and the dimensionless, orientation-averaged excluded volume $v_{\mathrm{ex}}$ for long-rods $[19,66]$. See Appendix E for the detailed definition of $v_{\mathrm{ex}}$ for different particle shapes. Analogously, the leading order term of the percolation threshold of overlapping particles of various shapes is also given by $1 / v_{\mathrm{ex}}$ [38]. In Fig. 2(a), compared with $\phi_{J}^{0}$ (filled), $\phi_{J}^{\infty}$ (open) remarkably collapses on a monotonically decreasing curve as a function of $v_{\text {ex }}$ for all the particle shapes studied here. This curve starts 
from the maximum $\phi_{J}^{\infty} \approx 0.56$ (i.e., the well-acknowledged RLP fraction), associated with the minimum $v_{\mathrm{ex}}=8$. We fit $\phi_{J}^{\infty}\left(v_{\mathrm{ex}}\right)$ by

$$
\phi_{J}^{\infty}=\frac{c_{1}}{v_{\mathrm{ex}}}-\frac{c_{2}}{v_{\mathrm{ex}}^{2}},
$$

where $c_{1}=7.868, c_{2}=27.157$ are the two fitting coefficients [dashed curve in Fig. 2(a)]. As explained in Appendix E, $c_{1}$ is the effective number of particles contained in the local excluded region of a central particle, and $c_{2}$ is associated with the correction from higher-order steric interactions among particles. Importantly, this universal behavior works well for general nonspherical particles, e.g., nonaxisymmetric ellipsoids and polyhedra, which demonstrates that $v_{\mathrm{ex}}$ plays a crucial role in determining $\phi_{J}^{\infty}$ for RLP compared to other shape parameters (e.g., aspect ratio). Therefore, one can accurately predict $\phi_{J}^{\infty}$ of arbitrary convex particle shapes simply based on the corresponding $v_{\mathrm{ex}}$, a local quantity easy to access. Additional simulations of frictional dimer particles as simple concave shapes [67] show that $z_{J}^{\infty} \approx 4$ and yet their $\phi_{J}^{\infty}$ are lower than the prediction of Eq. (1) (see Appendix F).

In the large asphericity limit $v_{\mathrm{ex}} \rightarrow \infty, \phi_{J}^{\infty} \sim v_{\mathrm{ex}}^{-1}$ asymptotically, reminiscent of the random packings of long-rods [19] and the percolation threshold of overlapping particles [38] as mentioned above. The latter system corresponds to a Poisson distribution of the overlapping particle centroids with random orientations. Here, the particles are impenetrable (i.e., at the jamming onset) in the RLPs yet their centroids can still possess almost random distribution when asphericity is sufficiently large. Accordingly, the shell structures in the packings, manifested as strong oscillations in the pair correlation function $g_{2}$, would be strongly suppressed for shapes with large asphericity, as shown in Appendix G, Fig. 8. The 2D schematic in Fig. 2(b) illustrates the difference of particle centroid correlations between a disk packing (the dashed circles indicate the shells of $g_{2}$ ) and an ellipse packing. Figure 2(b) further shows the translational order metric $T$ (see Appendix G) derived from the integral of $g_{2}$ in both MRJ and RLP states [68]. The abrupt decrease of $T$ as $v_{\mathrm{ex}}$ increases supports our speculations above.

Jammed packings with finite friction can be regarded as the interpolation between the MRJ and RLP states (see Appendix H). For a specific particle with finite $\mu$, we normalize $\phi_{J}(\mu)$ via $\tilde{\phi}(\mu)=\left(\phi_{J}(\mu)-\phi_{J}^{\infty}\right) /\left(\phi_{J}^{0}-\phi_{J}^{\infty}\right)$. In Fig. 2(c), we observe that $\tilde{\phi}(\mu)$ for all particle shapes shown in Fig. 1(j) again collapse on a master curve of $\mu$. Similar results for polyhedra in Fig. 1(k) are shown in Fig. 7(b). The empirical equation for this master curve is

$$
\tilde{\phi}=\frac{1}{1+\left(\mu / \mu^{*}\right)^{\alpha}},
$$

where $\alpha=1.07$, and $\mu^{*}=0.1635$. Note that $\alpha$ is very close to 1 , indicating the simple linear scaling behavior as the packing approaches the RLP, $\phi_{J}(\mu)-\phi_{J}^{\infty} \sim 1 / \mu$. The inset of Fig. 2(c) shows that $\phi_{J}(\mu)$ for sphere (squares with lines) becomes larger than the others for $\mu \gtrsim 3$, i.e., the monotonicity of $\phi_{J}$ with $v_{\text {ex }}$ only holds close to the RLP limit. This can be understood by the fact that the $\phi_{J}$ for nearly spherical particles is highly correlated with $z_{J}$, and $z_{J}$ for sphere is constantly smaller than the others with increasing $\mu$, until the
RLP limit is approached, as shown in Fig. 1(j). As discussed in Appendix $\mathrm{H}$, the normalized coordination number or the information about sliding contacts (see Appendix A) also displays similar universalities as a function of $\mu$ for different particles. From these results, the crossover $\mu^{*}=0.1635$ robustly marks the transition from frictionless to typical frictional packing behaviors.

For a specific particle shape, it is reasonable to expect that $\phi_{J}$ is positively correlated with $z_{J}$ as $\mu$ changes (see Appendix H, Fig. 10). In Fig. 2(d), the excess packing fraction $\phi_{J}-\phi_{J}^{\infty}$ exhibits a strong universal linear dependency on the excess coordination number $z_{J}-z_{J}^{\infty}$ for different particle shapes and $\mu$. This observation implies that $\phi_{J}(\mu)$ is mainly determined by two contributions: (i) $\phi_{J}^{\infty}$ determined by particle shape (i.e., $v_{\text {ex }}$ ) as the starting point, and (ii) an excess part linearly depending on $z_{J}$. Therefore, RLP could be regarded as a fundamental reference state to understand the full spectrum of frictional packings.

Here we note the effect of structural ordering on $\phi_{J}$ for certain nonspherical particles. In Fig. 2(a), $\phi_{J}^{0}$ of oblates (filled downward triangles) become larger than those of prolates (filled upward triangles) as $v_{\mathrm{ex}} \gtrsim 15$, as the consequence of finite local stacking ordering in oblate packings [Fig. 1(e)]. In Fig. 2(d), data of superball with $p=1.5$ (circles) and oblate with $w=0.2$ (open hexagons) deviate from the master curve also due to the ordering in these packings with small $\mu$.

\section{Percolation of contacting particle network as a precursor of jamming}

A jammed packing contains a system-spanning forcebearing backbone, involving the majority of the particles except for the rattlers. It can be expected that during the densification of the packing, particles aggregate, percolate, and eventually form a rigid backbone at the jamming point. Recent studies have reported the gap between the percolation transition and jamming transition for frictionless sphere (or disk) packings, indicating that a geometrically connected network, one unable to bear force, constitutes the precursor for the formation of the rigid backbone at jamming $[39,69]$. We expect that this percolation transition is related to RLP for different particle shapes.

We implement a depth-first search to trace the largest network formed by connected nonrattler particles for the intermediate unjammed configurations before jamming (see Appendix I). The spanning length of a network $L$ is calculated as the average over the three orthogonal directions. In Figs. 3(a)-3(d), we show the number fraction of particles involved in the largest network $\chi$ and its spanning length $L$ as a function of packing fraction $\phi$ for different particles. With increasing $\phi$ for $\mu=0.01,0.3$, and $10, \chi$ first slowly grows from $\sim 0$, then rapidly increases at intermediate $\phi$, and finally approaches $\sim 1$ close to $\phi_{J}(\mu)$, forming the rigid backbone at jamming. The increase of $\chi$ is rather sharp for $\mu=10$ and relatively smooth for $\mu=0.01$. The evolution of a network is also characterized by the increasing $L$. We can recognize a clear feature for percolation transition at $\phi_{P}$ where $\chi$ drastically rises and concurrently $L$ becomes 1 for the first time.

The vertical dashed lines, respectively, indicate $\phi_{J}^{0}$ and $\phi_{J}^{\infty}$ for the four different shapes. We also show $\phi_{P}$ and $\phi_{J}$ for 


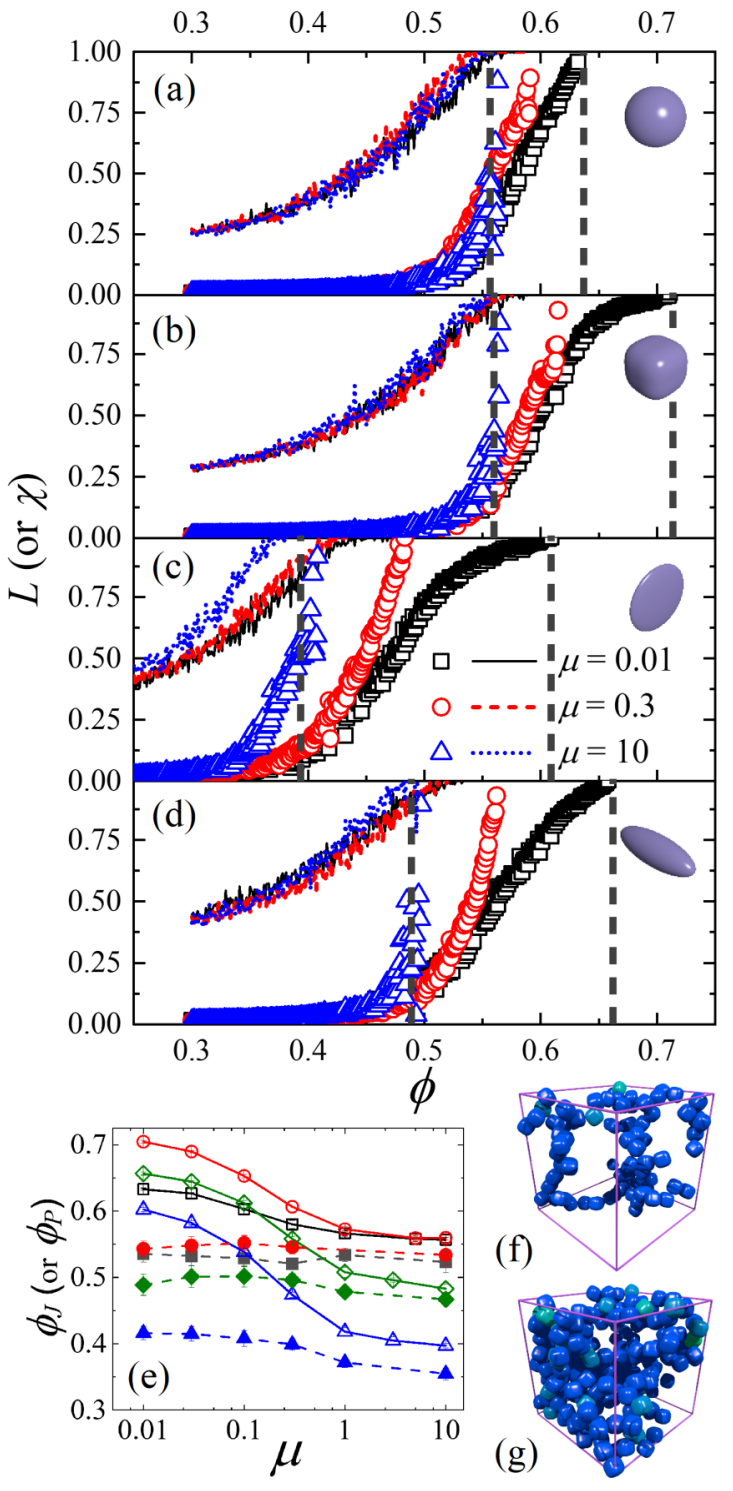

FIG. 3. Percolation analysis of contacting particle network during the densification process for four different particles. Evolution of the number fraction of particles involved in the largest network $\chi$ (scatters) and its spanning length $L$ (curves) during the increase of packing fraction $\phi$ for (a) sphere, (b) superball with $p=1.5$, (c) oblate with $w=0.2$, and (d) prolate with $w=3$. $\mu=0.01$, 0.3 , and 10 are considered. The vertical dashed lines indicate $\phi_{J}^{0}$ and $\phi_{J}^{\infty}$ for the four particles. (e) Packing fraction of percolation $\phi_{P}$ (filled) compared with $\phi_{J}$ (open) as a function of $\mu$ for the four particles (cubes, circles, diamonds, triangles in sequence). The above results are averaged over 20 independent realizations. Illustration of the largest network formation during densification for superball ( $p=$ 1.5) with $\mu=0.01$ : (f) $\phi=0.571, \chi=0.206$, and (g) $\phi=0.621$, $\chi=0.476$.

these shapes as a function of $\mu$ in Fig. 3(e). Interestingly, we observe that the gap between $\phi_{J}(\mu)$ and $\phi_{P}(\mu)$ reduces as $\mu$ increases, and importantly, $\phi_{P}(\mu) \approx \phi_{J}^{\infty}$ for all particle shapes and $\mu$. This implies that the nature of RLP can be understood as the percolation transition of the contacting particle network. For large $\mu$, such percolating network spontaneously gains rigidity at $\phi_{J} \gtrsim \phi_{P}$. Otherwise, all particles would rearrange collectively with different networks merged into the largest one during the densification, and the resulting jamming occurs at $\phi_{J}>\phi_{P}$. An example for this process is illustrated in Figs. 3(f) and 3(g). This explains how the finite structural correlations are introduced into the jammed packings with small $\mu$, at $\phi_{J}(\mu)>\phi_{J}^{\infty}$. Larger system sizes and more careful statistical analyses are needed to understand the criticality of such percolation transition more clearly.

\section{Density fluctuations in jammed frictional particle packings}

MRJ (frictionless) sphere packings are hyperuniform, manifested as the vanishing static structure factor in the zero wave-number limit [7]. In Fig. 4(a), we show the small wave-number regime of $S(K)$ ( $K$ is the spherically averaged, normalized wave number explained in Appendix $J$ ) for jammed sphere packings with $N=2.5 \times 10^{6}$ and different $\mu$. In the log-log plot, $S(K)$ decays as $K \rightarrow 0$ with different scalings varying from MRJ $(\mu=0)$ to $\operatorname{RLP}(\mu=50)$ states as $\mu$ increases. The short-dashed line indicates the linear scaling $S(K) \propto K$ for MRJ packings within the range $K \in[0.1,0.4]$, in agreement with previous studies [7,70]. The results with finite $\mu$ also exhibit a clear vestige of vanishing $S(K=0)$, indicating strong suppression of large-scale density fluctuations in these systems. Note that quantitatively similar $S(K)$ was also reported for jammed sphere packings with different $\mu$ at constant $\phi=0.64$ [71]. Table II in Appendix K shows the numerical extrapolation of $S(K)$ to the zero- $K$ limit for these packings. On the contrary, $S(K)$ at small $K$ is not suppressed for two intermediate unjammed configurations at $\phi=0.543$ and 0.592 during the densification. As an equivalent indicator to $S(K)$, the number density fluctuations $\left\langle\rho(l)^{2}\right\rangle-\langle\rho(l)\rangle^{2}$ for the same packings, where $\rho(l)$ is the particle number density in the window sphere with diameter $l$ (in the unit of particle diameter), are shown in Fig. 4(b). The number density fluctuation decays as $l^{-\gamma}$, where $\gamma=d+\beta$ with $0<\beta<1$ and $d=3$ in this work, reminiscent of the scalings reported in the critical absorbing states [72]. This behavior illustrates that the jammed frictional sphere packings are nearly hyperuniform [8].

For nonspherical packings, since the centroid cannot fully describe a particle, one should apply the spectral density $\tilde{\psi}(K)$ (see Appendix J) for a complete characterization of large-scale density fluctuations [43]. In Figs. 4(c) and 4(d), we show $\tilde{\psi}(K)$ of jammed packings of superball $(p=1.5)$ and prolate $(w=3)$ with $N=4000$ and varying $\mu$. Similar to frictional sphere packings, we observe the distinction between the vanishing $\tilde{\psi}(K)$ (see Table III in Appendix $\mathrm{K}$ ) and the significantly increasing $\tilde{\psi}(K)$ as $K \rightarrow 0$ for jammed (filled) and unjammed (open) configurations, respectively.

Like the MRJ packings for frictionless particles, we speculate that the origin of strong suppression of local density fluctuations in frictional packings also stems from the jamming nature of these systems $[3,7,29,43]$. For both frictionless and frictional particles, the requirement of jamming induces a well-defined topological contact network involving the majority of particles [see the evolution of network towards the jamming backbone in Figs. 3(a)-3(d)], which is crucial for establishing hyperuniformity. Similar geometrical and topological regulations like jamming could also be achieved via 

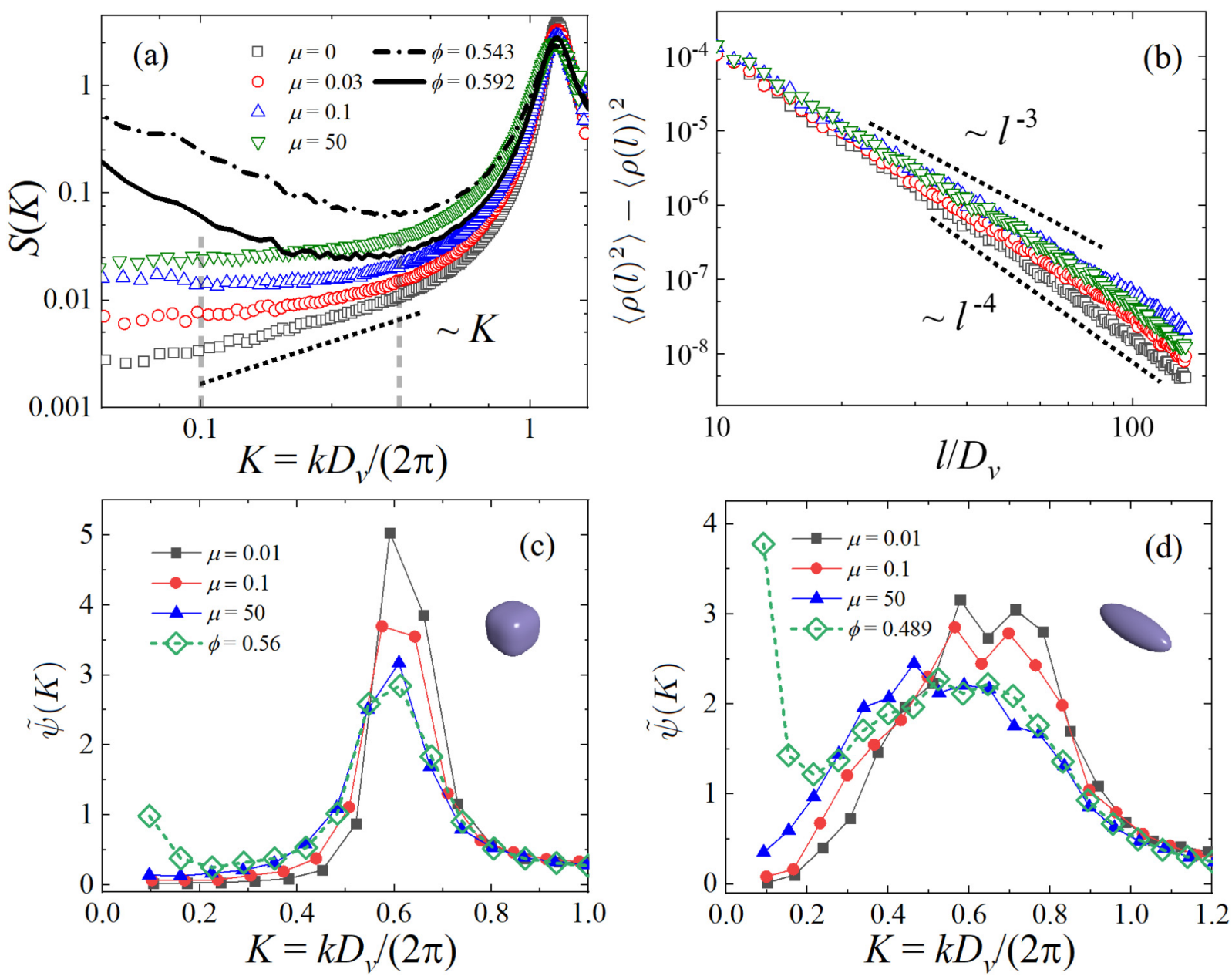

FIG. 4. Local density fluctuations of jammed frictional packings. (a) Static structure factor $S(K)$ and (b) number density fluctuation for different jammed sphere packings at $\phi_{J}(\mu)$, with $\mu=0,0.03,0.1$, and 50. In (a), $S(K)$ for two intermediate unjammed configurations of frictionless sphere at $\phi=0.543$ and 0.592 during the densification are indicated by the dash-dotted and solid curves, respectively. The shortdashed line indicates the scaling $S(K) \propto K$. The two vertical dashed lines indicate the interval $K \in[0.1,0.4]$. The two short-dashed lines in (b) indicate the scalings $l^{-3}$ and $l^{-4}$, respectively. Spectral density $\tilde{\psi}(K)$ for jammed packings of (c) superball with $p=1.5$ and (d) prolate with $w=3, \mu=0.01,0.1$, and 50. The dashed curves show the $\tilde{\psi}(K)$ for intermediate unjammed configurations for frictionless (c) superball at $\phi=0.56$ and (d) prolate at $\phi=0.489$, the same packing fractions as the corresponding RLPs.

other scenarios, such as the absorbing transition [72-74] or the optimization process of cellular structures [75]. We also note that the frictional packings are generally less dense than the corresponding frictionless packings, which can lead to larger local density fluctuations as observed here. Nonetheless, based on the numerical evidence in hands, we can reasonably infer that the disordered jammed packings of frictional particles are nearly hyperuniform $[7,29,43]$.

\section{CONCLUSIONS AND DISCUSSION}

This study advances our fundamental understanding of disordered jammed packings of frictional nonspherical particles. We discovered universal dependencies of fundamental packing characteristics (e.g., $\phi_{J}$ ) on particle shape (via dimensionless, orientation-averaged excluded volume $v_{\mathrm{ex}}$ ) and $\mu$. The RLP of nonspherical particles exhibits nearly constant $z_{J}^{\infty} \approx z_{\text {iso }}^{\infty}=4$ and minimally correlated local packing structures, making it an excellent reference state for understanding the entire spectrum of disordered jammed frictional packings. These features of RLP validate the rationale of a mean-field approximation based on $v_{\text {ex }}$. To be specific, for different particle shapes studied in this work, (i) $\phi_{J}^{\infty}$ of RLPs collapses on a monotonic decreasing function of $v_{\text {ex }}$ [Eq. (1)]; (ii) normalized packing fraction for finite $\mu$ is simply a function of $\mu$ [Eq. (2)]; (iii) $\phi_{J}(\mu)$ can be decomposed into $\phi_{J}^{\infty}$ as the starting point and an excess part linearly depending on $z_{J}$.

Furthermore, we showed that the frictional jamming transition is preceded by a percolation transition of contacting particle network, with an approximate relation $\phi_{P} \approx \phi_{J}^{\infty}$, illuminating the nature of RLP transition. Last but not least, we showed that the local density fluctuations are strongly suppressed on large length scales in jammed frictional packings, irrespective of particle shapes and $\mu$, indicating these packings are nearly hyperuniform.

Our results about static packing properties might provide insights on a number of complicated mechanical and dynamical phenomena in particulate systems, especially granular 
materials where friction is inevitable. For instance, particle shape significantly affects the mechanical strength and plastic events of granular materials [12,13]. Whether $\phi_{J}$ and $z_{J}$ as macroscopic or microscopic quantities can be leveraged to reveal the hidden correlations is not clear. Friction was found to play a key role in sheared granular materials [76,77]. The critical $\mu$ that marks the transition from caged to diffusive features is about $0.1-0.3$ [76], in general accordance with $\mu^{*}=0.1635$ shown in Fig. 2(c). This might not be a simple coincidence and needs further exploration.

\section{ACKNOWLEDGMENTS}

Y.J. thanks Arizona State University for support and Peking University for hospitality during his sabbatical leave. Y.Y. and S.L. acknowledge support from the National Natural Science Foundation of China (No. 11972047 and No. 11572004) and the High-Performance Computing Platform of Peking University.

\section{APPENDIX A: SIMULATION DETAILS}

We implement MD simulations with the linear springdashpot model and static friction to generate packing configurations [58,59]. For superellipsoids, we apply the Perram and Wertheim potential to check the overlap between two particles $[18,48]$. For neighboring superellipsoids $i$ and $j$, we obtain the factor $\eta_{i j}$ to rescale their axes that brings them to exact tangency, as well as the tangency position (contact point) and its normal direction. Particles $i, j$ overlap when $\eta_{i j}<1$ and the potential energy is

$$
U_{i j}=\frac{1}{2} \epsilon\left(1-\eta_{i j}\right)^{2},
$$

where $\epsilon$ is the energy scale. The corresponding repulsive elastic force from particle $j$ to particle $i$ is

$$
\vec{f}_{i j}^{e}=\frac{\epsilon \eta_{i j}\left(1-\eta_{i j}\right) \hat{n}_{i j}}{\vec{r}_{i j} \cdot \hat{n}_{i j}},
$$

where $\vec{r}_{i j}$ and $\hat{n}_{i j}$ are the center-to-center vector and the unit normal of the tangency point, respectively.

The contact-force constitutive relation for polyhedral particles is relatively ambiguous due to the different contact types formed by vertexes, edges, and facets. Here we implement the so-called "spheropolyhedral" model to avoid the complex polyhedral intersection computation [30,31]. A spheropolyhedron is created by sweeping the profile of an ideal polyhedron using a sphere with diameter $h_{s}$. $h_{s}$ quantifies the thickness of the rounded shell and we set $h_{s}=0.01 L_{e}$ to approximate an ideal polyhedron, where $L_{e}$ is the characteristic length chosen to be the edge length for Platonic solids or $\sqrt[3]{a_{1} a_{2} a_{3}}$ for cuboids with side lengths $a_{1}, a_{2}, a_{3}$. For neighboring polyhedra $i$ and $j$, we calculate the distance vectors for all the vertex-facet and edge-edge pairs. If any distance $d_{i j}^{k}$ is less than $2 h_{s}$, particles $i, j$ overlap. There might be multiple contacts between two polyhedra, which explicitly reflects the complex contact topology. In this work, for a contacting particle pair, the number of such a multiple contact would be dominantly 1,2 , or 3 (with a negligible part larger than 3 that is counted as 3 here), which corresponds to the different contact topologies, i.e., point contact (P), edge-facet contact
(E-F), or facet-facet contact (F-F), respectively. On average, the summation of multiple contact numbers over all the contacts gives the constraint number $C$ for a particle,

$$
C=z_{J}\left(f_{c}(P)+2 f_{c}(E F)+3 f_{c}(F F)\right),
$$

where $f_{c}$ quantifies the relative fraction of a contact type within a packing. Obviously, $C \geqslant z_{J}$.

The associated potential energy for two contacting polyhedra is then

$$
U_{i j}=\frac{\epsilon}{2 D_{v}^{2}} \Sigma_{k}\left(d_{i j}^{k}-2 h_{s}\right)^{2},
$$

where $\mathrm{k}$ loops over all vertex-facet or edge-edge pairs that lead to overlaps. The joint elastic force is

$$
\vec{f}_{i j}^{e}=\Sigma_{k} \vec{f}_{i j}^{e, k}=\frac{\epsilon}{D_{v}^{2}} \Sigma_{k}\left(2 h_{s}-d_{i j}^{k}\right) \hat{d}_{i j}^{k},
$$

where $\hat{d}_{i j}^{k}$ is the direction of the $k$ th distance vector.

The subsequent tangential forces and dissipative forces in both normal and tangential directions are introduced using the conventional formula [59]. The tangential elastic constant is $0.5 \epsilon / D_{v}^{2}$. Both the normal and tangential restitution coefficients are 0.1 , which determine the associated normal and tangential damping constants. The characteristic time scale is $t_{0}=D_{v} \sqrt{\epsilon / m}$ where $m$ is the particle mass and we set the simulation time step $\Delta t=0.01 t_{0} \sim 0.05 t_{0}$. The static friction coefficient $\mu$ is imposed by the constraint $F_{t} \leqslant \mu F_{n}$, where $F_{t}$, $F_{n}$ are the magnitudes of total tangential and normal forces (including both the elastic and dissipative parts) for a specific contact. A contact is termed "sliding" if the truncation $F_{t}=$ $\mu F_{n}$ works. Note that normal forces would introduce torques for a general nonspherical particle. We use the velocity-Verlet scheme to integrate particle translational moves, and the extended leapfrog scheme for particle rotation [58]. The moment of inertia for a specific particle is calculated analytically or numerically.

The quasistatic compression protocol is as follows. In a periodic cubic cell, we randomly initialize an overlap-free dilute configuration of $N$ particles at packing fraction $\phi \lesssim 0.1$. The system is isotropically compressed, i.e., both the boundary length and all the particle centroid coordinates are rescaled by a factor less than 1, with the pre-set packing fraction increment $\delta \phi=0.0005-0.001$, followed by constant volume MD. During the MD process, we frequently check the total potential energy $U=\Sigma_{i<j} U_{i j}$ and the total kinetic energy $U^{\prime}$. If $U / N<U_{\text {tol }}$, where $U_{\text {tol }}=10^{-10} \epsilon-10^{-8} \epsilon$ is the jamming threshold, the system is unjammed and the isotropic compression is repeated. Otherwise, $U^{\prime}$ will vanish with finite $U$. If $U^{\prime}<5 \times 10^{-5} U$, we further check the mechanical equilibrium by evaluating the ratio between the average contact force magnitude (including torque) and the joint force magnitude for each particle. Once this value is sufficiently small, we obtain a jammed packing slightly above the exact jamming onset, with the distance $\Delta \phi$ bounded by both $U_{\text {tol }} / \epsilon$ and $\delta \phi$, $10^{-5} \lesssim \Delta \phi \lesssim 10^{-3}$. For the linear spring model here, the order of magnitude of system pressure is $P D_{v}^{3} / \epsilon \sim \Delta \phi$. This procedure is the extension of the original quasistatic compression algorithm to generate jammed packings for frictionless soft sphere [57] and also implemented for frictional sphere [35]. Here MD simulation shows certain numerical instability 

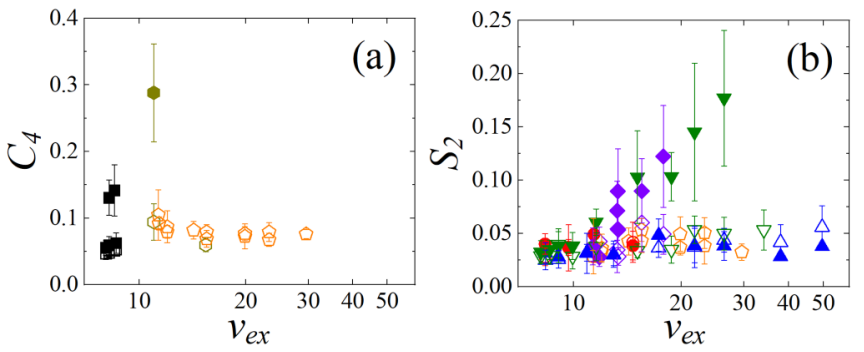

FIG. 5. (a) Cubatic order $C_{4}$ and (b) nematic order $S_{2}$ in both the MRJ (filled) and RLP (open) states for all the particles. Data symbols are the same as Fig. 1(i).

for polyhedra $\mu=0$. But $\mu=0.01$ notably improves such issue and jammed polyhedral packings with small $\mu$ can also reasonably approach the frictionless limit [see Appendix D, Fig. 7(b)]. In addition, we implement the same protocol with L-BFGS energy minimization for frictionless superellipsoids [18], which accords with the MD method with $\mu=0$.

Note that mechanical equilibrium is evaluated after excluding rattlers. In the extreme case, two contacts are the minimal requirement to stabilize a frictional particle. Therefore, we recursively count the contact number for each particle $z_{i}$ after excluding rattlers with $z_{i}<2$. The coordination number is $z_{J}=\left\langle z_{i}\right\rangle$ after excluding rattlers.

\section{APPENDIX B: ORIENTATIONAL ORDER METRICS}

We calculate two orientational order metrics [18], namely cubatic order $C_{4}$ and nematic order $S_{2}$, for both the MRJ and RLP states, shown in Fig. 5. MRJ packings for certain cubelike superballs ( $p=1.5$ and 2) possess $C_{4}>0.1$ and even $C_{4} \approx 0.3$ for the cube (filled hexagon). $C_{4} \lesssim 0.1$ for all the RLPs. As for $S_{2}$, MRJ packings for oblates (filled
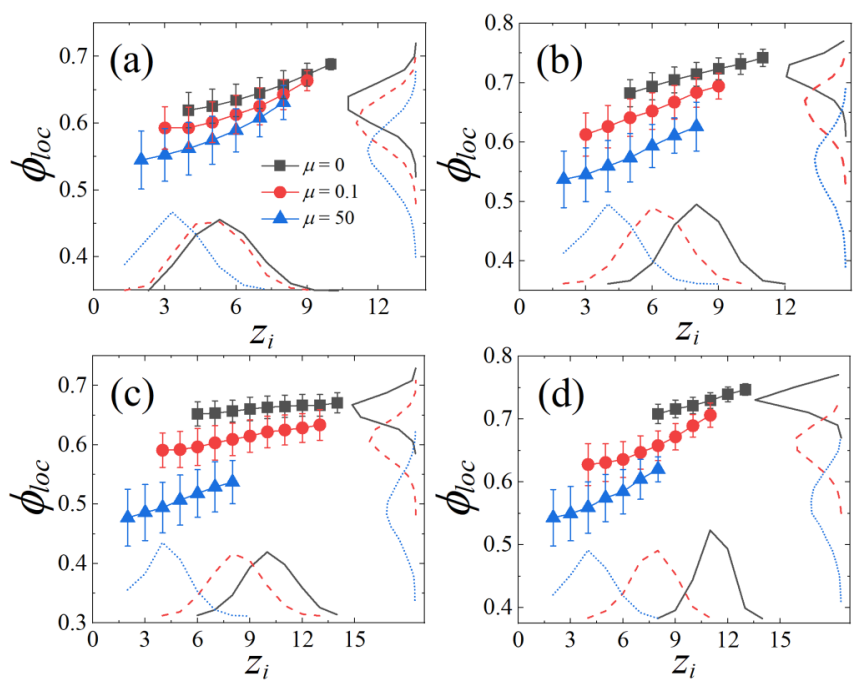

FIG. 6. Relation between local packing fraction $\phi_{\text {loc }}$ and local contact number $z_{i}$ in packings for (a) sphere, (b) superball with $p=1.5$, (c) prolate with $w=3$, and (d) self-dual ellipsoid with $w_{1}=0.8, \mu=0,0.1$, and 50. The associated distributions of $\phi_{\mathrm{loc}}$ and $z_{i}$ are also shown along the vertical and horizonal axes, respectively.
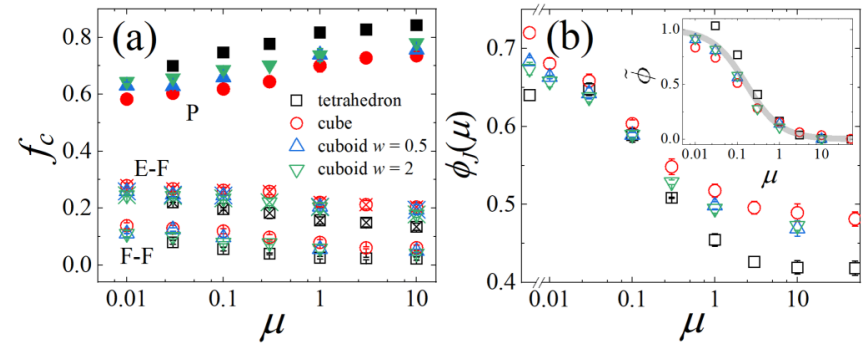

FIG. 7. (a) Relative fraction $f_{c}$ of point (P), edge-facet (E-F), and facet-facet (E-F) contacts in jammed packings for four polyhedra, including tetrahedron, cube, and two cuboids, with different $\mu$. (b) Jamming packing fraction $\phi_{J}(\mu)$ as a function of $\mu$ for these polyhedra. The leftmost data indicate the previous numerical results of frictionless packings [45,52-54]. The inset in (b) shows the normalized packing fraction $\tilde{\phi}$ and the empirical Eq. (2) is indicated by the thick curve.

downwards triangles) or several general ellipsoids close to oblates (filled diamonds) possess relatively large $S_{2}$, while all the others remain $S_{2} \lesssim 0.05$. These observations validate that RLPs for all the particles are constantly random compared to the frictionless cases (MRJ).

\section{APPENDIX C: LOCAL PACKING PROPERTIES}

We implement the set Voronoi tessellation method [49] to measure the local packing fraction $\phi_{\text {loc }}$ for each individual particle in a packing, defined by the ratio between particle volume and the associated Voronoi cell volume. In Fig. 6, we show the average $\phi_{\text {loc }}$ binned at different local contact number $z_{i}$ for four particle shapes with $\mu=0,0.1$, and 50. Clearly, the relation between $\phi_{\mathrm{loc}}$ and $z_{i}$ is not universal.

\section{APPENDIX D: PACKINGS OF POLYHEDRA}

Our spheropolyhedral approximation for ideal polyhedra gives the multiple contacts between two polyhedra (see Appendix A), which explicitly reflects the complex contact topologies, i.e., point, edge-facet, and facet-facet contacts. As shown in Fig. 7(a), the fractions of three types of contacts show similar dependencies on $\mu$ for four polyhedra, where the point contacts dominate and the facet-facet contacts account for $\lesssim 0.15$.

$\phi_{J}(\mu)$ as a function of $\mu$ for these four polyhedra is shown in Fig. 7(b), which is similar to Fig. 2 for superellipsoids. Specifically, with vanishing $\mu, \phi_{J}(\mu)$ for polyhedra using our model indeed approaches the frictionless limit from previous simulations using ideal polyhedral model (leftmost data), i.e., $\phi_{J}^{0} \approx 0.64$ for tetrahedron $[45,52,53], \phi_{J}^{0} \approx 0.72$ for cube $[45,52], \phi_{J}^{0} \approx 0.68$ or 0.67 for cuboids with $w=0.5$ or 2 [54].

\section{APPENDIX E: EXCLUDED VOLUME}

Consider a test particle of centroid position $\vec{r}$ and orientation $\vec{\omega}$ with respect to a fixed particle (of identical shape), the indicator function $f_{\mathrm{ex}}(\vec{r}, \vec{\omega})=1$ when the two particles overlap and otherwise $f_{\mathrm{ex}}(\vec{r}, \vec{\omega})=0$. The dimensionless, 
TABLE I. Analytical results of dimensionless, orientation-averaged excluded volume $v_{\mathrm{ex}}$.

\begin{tabular}{lcc}
\hline \hline Particle shape & Description & $v_{\text {ex }}$ \\
\hline Sphere & - & 8 \\
Tetrahedron & - & $2+\frac{18}{\pi} \sqrt{\frac{3}{2}} \cos ^{-1}\left(-\frac{1}{3}\right)$ \\
Cube & - & 11 \\
Cuboid & Sides $a_{1}, a_{2}, a_{3}$ & $2+\frac{\left(\Sigma_{i<j}^{3} a_{i} a_{j}\right)\left(\Sigma_{i=1}^{3} a_{i}\right)}{a_{1} a_{2} a_{3}}$ \\
Prolate & Aspect ratio $w>1, e^{2}=1-1 / w^{2}$ & $2+\frac{3}{2}\left(1+\frac{w}{e} \sin ^{-1} e\right)\left(1+\frac{1}{w^{2} e} \tanh ^{-1} e\right)$ \\
Oblate & Aspect ratio $w<1, e^{2}=1-w^{2}$ & $2+\frac{3}{2}\left(1+\frac{w^{2}}{e} \tanh ^{-1} e\right)\left(1+\frac{1}{w e} \sin ^{-1} e\right)$ \\
\hline \hline
\end{tabular}

orientation-averaged excluded volume is then defined by

$$
v_{\mathrm{ex}}=V_{p}^{-1} \iint f_{\mathrm{ex}}(\vec{r}, \vec{\omega}) d \vec{r} d \vec{\omega},
$$

where $V_{p}$ is the particle volume. Apparently, $v_{\mathrm{ex}}=2^{3}=8$ for sphere. In Table I, we show the analytical results of $v_{\text {ex }}$ for a part of particles considered in this work [38].

For superballs and nonaxisymmetric ellipsoids, we calculate $v_{\mathrm{ex}}$ numerically. First, a particle with unit volume is fixed at the origin centroid. Then, we uniformly sample an identical test particle with (i) $\vec{\omega}$ in all spatial orientations and (ii) centroid position $\vec{r}$ within a cubic space of length $5 D_{v}-10 D_{v}$ centered at the origin. This sample is repeated $5 \times 10^{9}-1 \times 10^{10}$ times. As a result, the ratio between the sample count when the test particle overlaps with the fixed one and the total count, multiplied by the cubic volume gives the $v_{\text {ex }}$ for this specific shape.

Considering the minimal correlations among particles for the RLPs, the prerequisite $z_{J}=4$ allows us to use the following mean-field approximation for the local contact density in a region containing a central particle and its neighbors,

$$
c_{1}\left(z_{J}\right)=\iint \rho(\vec{r}, \vec{\omega}) f_{\mathrm{ex}}(\vec{r}, \vec{\omega}) d \vec{r} d \vec{\omega},
$$

where $\rho$ is the particle number density. Assume $\rho$ is a meanfield constant, which can be taken outside the integral, and apply the definition of $v_{\mathrm{ex}}$, then we have $c_{1}\left(z_{J}\right)=\phi v_{\mathrm{ex}}$, where $\phi=\rho V_{p}$ corresponds to $\phi_{J}^{\infty}$. For RLPs of nearly spherical particles with nonvanishing short-range pair correlations, this first-order approximation does not hold and the second-order correction $c_{2}$ can be introduced, which leads to Eq. (1) in the main text.

\section{APPENDIX F: EFFECT OF PARTICLE CONCAVITY}

We generate RLPs of different dimer particles with $N=$ $500, \mu=10$, and dimer aspect ratio $w=1.1,1.25$, and 1.5, whose contact forces are defined separately for the two element spheres not including approximation like polyhedra. $z_{J}^{\infty}=4$ holds with high accuracy for these packings and the fraction of multiple contacts, i.e., two dimers interacting through more than one sphere-to-sphere contact, is notably small in contrast to the frictionless case [67]. $v_{\mathrm{ex}}$ for these three dimers are numerically calculated as $8.03,8.17$, and 8.65 , with the fitted $\phi_{J}^{\infty}=0.558,0.556$, and 0.546 using Eq. 1, respectively. The simulated $\phi_{J}^{\infty}=0.552,0.550$, and 0.527 are lower than the fitting. Whether or not considering the forbidden volume of dimers (i.e., region around the junc- tion of two spheres inaccessible to any other dimer particles) does not affect this result. Therefore, we are inclined to restrict the application of Eq. 1 for convex shapes.

\section{APPENDIX G: PAIR CORRELATION FUNCTION AND TRANSLATIONAL ORDER METRIC}

In Fig. 8, we show the pair correlation function $g_{2}$ in jammed packings for different particle shapes and $\mu$. All the peaks of $g_{2}$ are tamed to some extent for RLPs. For sufficiently aspherical particles, such as prolate with $w=3$ and oblate with $w=0.3, g_{2}$ behaves similarly in frictionless packings or RLPs, with the strong oscillation suppressed.

We apply the translational order metric $T$ derived from the integral of $g_{2}$ as follows [68]:

$$
T=\frac{\int_{r_{\min }}^{r_{\max }}\left|g_{2}(r)-1\right| d r}{r_{\max }-r_{\min }},
$$

where $r_{\min }$ is the minimal distance when $g_{2}$ becomes nonzero, $r_{\max }=3 D_{v}$. From Fig. $2(\mathrm{~b})$, we find that $T \lesssim 0.4$ for nearly spherical particles in agreement with previous studies $[29,68]$ and $T$ drastically decreases as particle asphericity increases ( $v_{\text {ex }}$ grows accordingly).
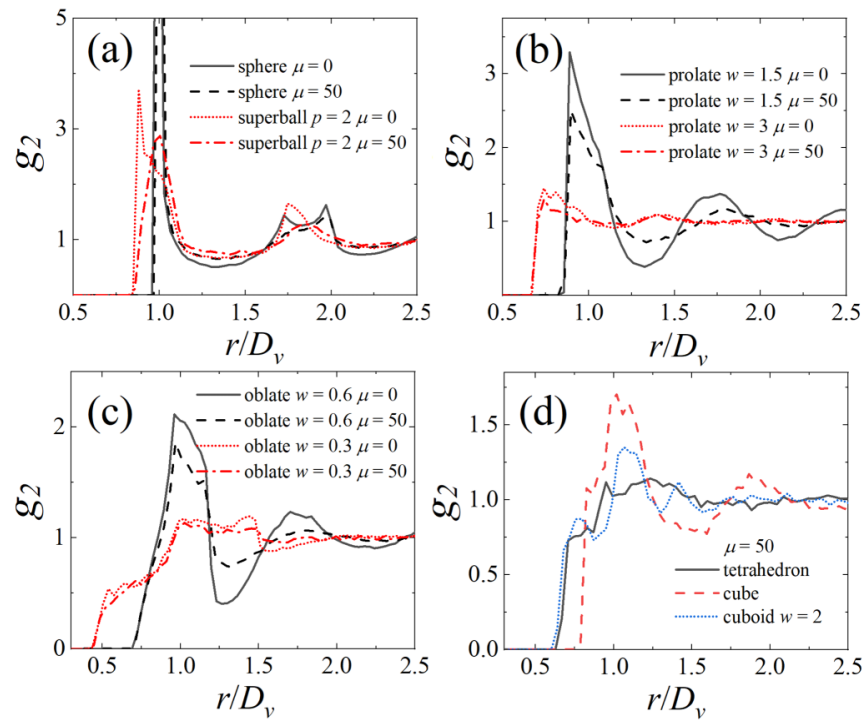

FIG. 8. Pair correlation function $g_{2}$ in jammed packings for (a) sphere and superball, (b) prolates, (c) oblates, and (d) polyhedra, with different $\mu$. 

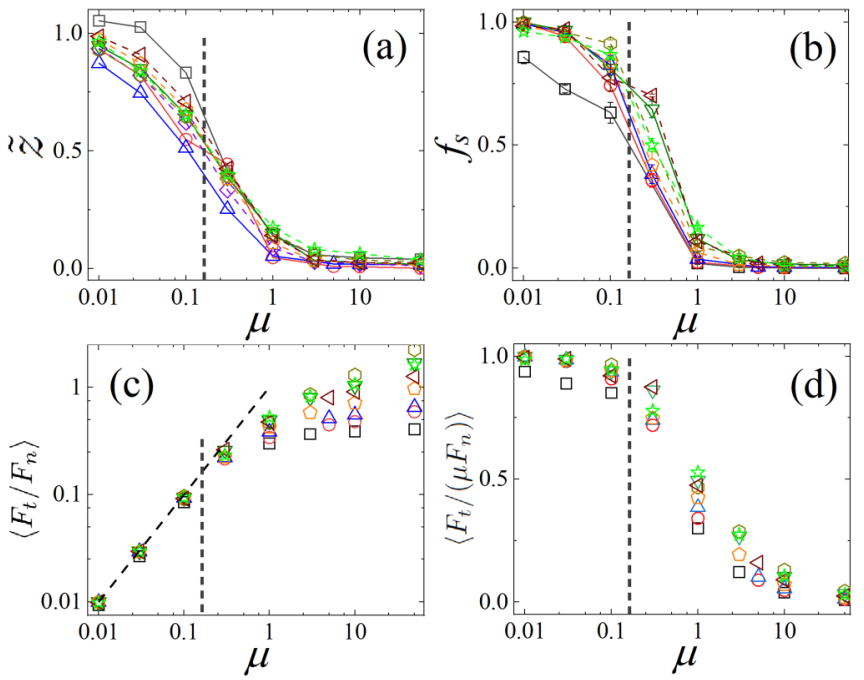

FIG. 9. (a) Normalized coordination number $\tilde{z}$ [Eq. (H2)], (b) fraction of sliding contacts $f_{s}$, (c) average ratio between magnitudes of tangential and normal contact forces $\left\langle F_{t} / F_{n}\right\rangle$, and (d) $\left\langle F_{t} /\left(\mu F_{n}\right)\right\rangle$ as functions of $\mu$ for different particles shown in Fig. 1(j) (the same symbols). The vertical dashed lines indicate the crossover friction coefficient $\mu^{*}=0.1635$. Another dashed line in (c) has the unit slope crossing the origin.

\section{APPENDIX H: OTHER UNIVERSALITIES}

Without loss of generality, we can express the packing fraction $\phi_{J}(\mu)$ with finite $\mu$ as

$$
\phi_{J}(\mu)=A(\mu) \phi_{J}^{0}+(1-A(\mu)) \phi_{J}^{\infty},
$$

where $A(\mu)$ is an interpolation index that satisfies $A(\mu=$ $0)=1$ and $A(\mu \rightarrow \infty)=0$. The simplest mathematical form is $A(\mu)=\mu^{*} /\left(\mu^{*}+\mu\right)$, where $\mu^{*}$ is a constant. Substituting this expression for $A(\mu)$ into Eq. (H1) yields $\tilde{\phi}(\mu)=$ $\left(\phi_{J}(\mu)-\phi_{J}^{\infty}\right) /\left(\phi_{J}^{0}-\phi_{J}^{\infty}\right)=\left[1+\mu / \mu^{*}\right]^{-1}$. Equation (2) in the main text replaces $\mu / \mu^{*}$ with $\left(\mu / \mu^{*}\right)^{\alpha}$ as the generalized form and the exponent $\alpha=1.07$ is indeed close to 1 .
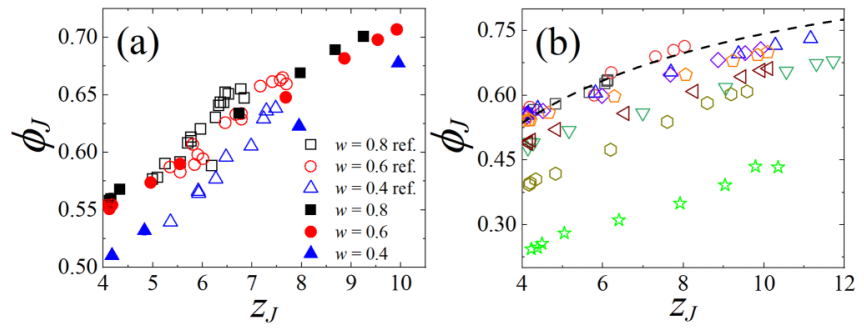

FIG. 10. Relation between jamming packing fraction $\phi_{J}$ and coordination number $z_{J}$ for (a) three oblates with $w=0.8,0.6$, and 0.4, and (b) different particles shown in Fig. 1(j) (the same symbols). In (a), we show the results from a previous experiment (open) [49] for comparison. The dashed curve in (b) indicates the relation $\phi_{J}=z_{J} /\left(2 \sqrt{3}+z_{J}\right)$ originally proposed for sphere [23].
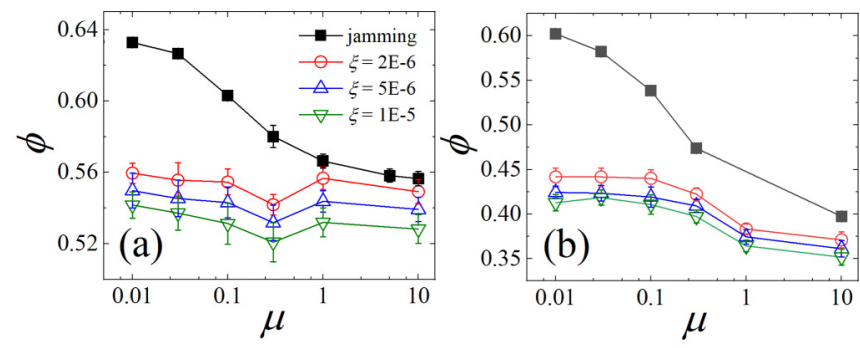

FIG. 11. The packing fraction of percolation $\phi_{P}$ (open) based on different contact cutoff $\xi$ compared with $\phi_{J}$ (filled) as a function of $\mu$ for (a) sphere and (b) oblate with $w=0.2$. We define two superellipsoids to be contacted when their rescaling factor $\eta_{i j}^{2}<1+\xi$, where $\xi>0$ is a tiny cutoff value (see Appendix A). The trend of $\phi_{P}$ does not change with varying $\xi$. We choose $\xi=5 \times 10^{-6}$ for the percolation analysis.

Besides packing fraction, we find that the normalized coordination number, defined by

$$
\tilde{z}(\mu)=\frac{z_{J}(\mu)-z_{J}^{\infty}}{z_{J}^{0}-z_{J}^{\infty}},
$$

shows similar dependency on $\mu$ for different particles with the same crossover $\mu^{*}=0.1635$, as shown in Fig. 9(a). Moreover, Fig. 9(b) indicates that the decay of sliding contacts with increasing $\mu$ shares the same feature as both $\tilde{\phi}$ and $\tilde{z}$. $F_{t}=\mu F_{n}$ nearly holds for $\mu<\mu^{*}$, and there exists a distribution $0 \leqslant F_{t} \leqslant \mu F_{n}$ for larger $\mu$, as validated by Figs. 9(c) and 9 (d). The same phenomenon was previously reported for frictional sphere packings [35]. Here we observe the unexpected universalities among different particles.

Actually, the similar dependencies of $\tilde{\phi}$ and $\tilde{z}$ on $\mu$ can be regarded as the consequence of the simple linear relation $\phi_{J}-\phi_{J}^{\infty} \propto z_{J}-z_{J}^{\infty}$ for different particle shapes. In Fig. 10, we show $\phi_{J}$ versus $z_{J}$ for different frictional particles in comparison with a previous experiment for oblates [49]. For all the particles, $\phi_{J}$ approximately demonstrates a linear dependency
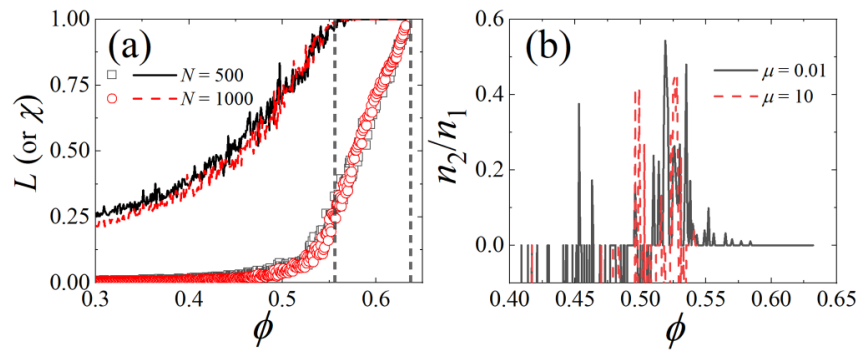

FIG. 12. (a) The number fraction of particles involved in the largest network $\chi$ (symbols) and its spanning length $L$ (curves) as a function of $\phi$ for sphere with $\mu=0.01, N=500$ or 1000 . The results are averaged over 20 independent realizations. Vertical dashed lines indicate $\phi_{J}^{\infty}$ and $\phi_{J}^{0}$. (b) The ratio between the number of nonrattler particles excluding those within the largest network $n_{2}$ and the particle number within it $n_{1}$ as a function of $\phi$ for sphere with $\mu=0.01$ and $10, N=1000$. We set $n_{2} / n_{1}<0$ when all the particles are rattlers and $n_{2} / n_{1}=0$ means there is only one network. Here we show two specific configurations. 
TABLE II. Fitting results of structure factor $S(K)$ in the small- $K$ regime for jammed packings of frictional spheres.

\begin{tabular}{lllll}
\hline \hline Term & \multicolumn{1}{c}{$\mu=0$} & $\mu=0.03$ & $\mu=0.1$ & $\mu=50$ \\
\hline$\beta$ & 0.982 & 0.847 & 0.274 & 0.218 \\
$S_{0}$ & 0.000908 & 0.00302 & -0.000989 & -0.000673 \\
\hline
\end{tabular}

on $z_{J}$ and even shares a common slope. This finding leads to the collapse in Fig. 2(d).

\section{APPENDIX I: CONTACTING NETWORK ANALYSIS}

It is subtle to define contacts for an unjammed configuration because all the particles in such a packing can collectively rearrange by a tiny magnitude to erase all the interparticle overlaps. In our MD simulation, we cease a particle's movements (both velocity and angular velocity are set to zero) if it does not overlap with the others. Consequently, a number of particles will touch the others although they are not force balanced [69]. As mentioned above, rattlers need to be detected frequently before the mechanical equilibrium criterion being checked. Different from the precise contact detection, here we soften the contact criterion to define the contacting particle network geometrically including those nearly touching particles, as shown in Fig. 11. Afterwards, we perform the same recursive method to detect rattlers. Then, a depth-first search is implemented to trace the largest network formed by connected nonrattler particles. During the quasistatic compression procedure, we analyze the network for the transient unjammed packings $\left(U / N<U_{\text {tol }}\right)$ before the next isotropic compression.

We define the domain of particle $i$ in the $x$ direction as $\left[X_{i}-0.5 D_{v}, X_{i}+0.5 D_{v}\right]$, where $X_{i}$ is the $x$ coordinate of its centroid and $D_{v}$ is its size. The covering fraction of the union of all the particle domains within a network projected upon the boundary in the $x$ direction is $L_{x}$ and similarly for $L_{y}$ and $L_{z}$ (periodic boundary condition is properly considered). Thus, the spanning length of a network is the average over the three directions $L=\sqrt{\left(L_{x}^{2}+L_{y}^{2}+L_{z}^{2}\right) / 3} . L=1$ means percolation. We judge a percolation when $L>0.99$ in three successive steps of $\phi$, which ensures a stable percolated network. Finite size effect is negligible for such analysis shown in Fig. 12(a). In Fig. 12(b), we notice the instability of the existence of a network for $\phi<\phi_{P}$ and the formation of a single systemspanning network $\left(n_{2} / n_{1}=0\right)$ for $\phi>\phi_{P}, \mu=0.01$.

\section{APPENDIX J: SPECTRAL DENSITY}

For a given packing, the spectral density $\tilde{\psi}(\vec{k})$ is expressed as

$$
\tilde{\psi}(\vec{k})=\frac{1}{C^{3}}\left|\Sigma_{j=1}^{N} e^{-i \vec{k} \cdot \vec{r}_{j}} \hat{M}\left(\vec{k} ; \tilde{p}_{j}\right)\right|^{2},
$$

where $C$ is the side length of the cubic simulation cell, $\vec{k}=$ $(2 \pi / C) \vec{z}$ with $\vec{z} \in \mathbb{Z}^{3}$ is the wave vector, $\vec{r}_{j}$ is the centroid position of particle $j$, and $\hat{M}\left(\vec{k} ; \tilde{p}_{j}\right)$ is the Fourier transform of the indicator function for particle $j$ whose position and orientation are represented by $\tilde{p}_{j}$ [43]. Specifically,

$$
\hat{M}\left(\vec{k} ; \tilde{p}_{j}\right)=\int e^{-i \vec{k} \cdot \vec{r}} M\left(\vec{r} ; \tilde{p}_{j}\right) d \vec{r},
$$

where the indicator function $M\left(\vec{r} ; \tilde{p}_{j}\right)=1$ if $\vec{r}$ locates inside particle $j$ and otherwise $M=0$. We numerically calculate $\hat{M}$ by discretizing the space around a given particle into pixels. Due to the radial symmetry of disordered packings, $\tilde{\psi}(\vec{k})$ with the vector $\vec{k}$ inside a spherical shell of thickness $2 \pi / C$ are averaged to obtain $\tilde{\psi}(k)$. Further, we show the results in units of $K=k D_{v} /(2 \pi)$ in the main text.

\section{APPENDIX K: EXTRAPOLATION FOR SMALL-K REGIME}

For jammed packings of frictional sphere, we fit $S(K)$ for $K \in[0.1,0.4]$ using the equation $S(K)=S_{0}+a K^{\beta}$, where $\beta$ is the slope of the log-log plot [Fig. 4(a)], as shown in Table II. We check that altering the specific fitting equation does not change the magnitude of $S_{0}$ much. In spite of the limited numerical precision, the small- $K$ behaviors of $S(K)$ in jammed packings are distinct from those in unjammed packings with the same packing fractions.

Similarly, for nonspherical packings shown in Figs. 4(c) and $4(\mathrm{~d})$, we perform polynomial fitting for $K \lesssim 0.4$ to obtain the zero- $K$ extrapolation $\tilde{\psi}_{0}$, as shown in Table III. We note the moderate system size for nonspherical packings $N=$ 4000 , such that $\tilde{\psi}_{0}$ is not as close to 0 as $S_{0}$.

TABLE III. Fitting results of spectral density $\tilde{\psi}(K)$ in the small$K$ regime for jammed packings of frictional nonspherical particles.

\begin{tabular}{lcccr}
\hline \hline Particle shape & Term & $\mu=0.01$ & $\mu=0.1$ & $\mu=50$ \\
\hline Superball $(p=1.5)$ & $\tilde{\psi}_{0}$ & -0.012 & 0.002 & 0.007 \\
Prolate $(w=3)$ & $\tilde{\psi}_{0}$ & -0.004 & -0.04 & -0.01 \\
\hline \hline
\end{tabular}

[1] M. van Hecke, Jamming of soft particles: geometry, mechanics, scaling and isostaticity, J. Phys.: Condens. Matter 22, 033101 (2009).

[2] A. J. Liu and S. R. Nagel, The jamming transition and the marginally jammed solid, Annu. Rev. Condens. Matter Phys. 1, 347 (2010).

[3] S. Torquato and F. H. Stillinger, Jammed hard-particle packings: From Kepler to Bernal and beyond, Rev. Mod. Phys. 82, 2633 (2010).
[4] A. Baule, F. Morone, H. J. Herrmann, and H. A. Makse, Edwards statistical mechanics for jammed granular matter, Rev. Mod. Phys. 90, 015006 (2018).

[5] J. Bernal and J. Mason, Packing of spheres: co-ordination of randomly packed spheres, Nature (London) 188, 910 (1960).

[6] G. Parisi and F. Zamponi, Mean-field theory of hard sphere glasses and jamming, Rev. Mod. Phys. 82, 789 (2010). 
[7] A. Donev, F. H. Stillinger, and S. Torquato, Unexpected Density Fluctuations in Jammed Disordered Sphere Packings, Phys. Rev. Lett. 95, 090604 (2005).

[8] S. Torquato, Hyperuniformity and its generalizations, Phys. Rev. E 94, 022122 (2016).

[9] A. Baule, R. Mari, L. Bo, L. Portal, and H. A. Makse, Meanfield theory of random close packings of axisymmetric particles, Nat. Commun. 4, 2194 (2013).

[10] A. Donev, I. Cisse, D. Sachs, E. A. Variano, F. H. Stillinger, R. Connelly, S. Torquato, and P. M. Chaikin, Improving the density of jammed disordered packings using ellipsoids, Science 303, 990 (2004).

[11] Y. Kallus, The random packing density of nearly spherical particles, Soft Matter 12, 4123 (2016).

[12] A. G. Athanassiadis, M. Z. Miskin, P. Kaplan, N. Rodenberg, S. H. Lee, J. Merritt, E. Brown, J. Amend, H. Lipson, and H. M. Jaeger, Particle shape effects on the stress response of granular packings, Soft Matter 10, 48 (2014).

[13] K. A. Murphy, K. A. Dahmen, and H. M. Jaeger, Transforming Mesoscale Granular Plasticity Through Particle Shape, Phys. Rev. X 9, 011014 (2019).

[14] Z. Zheng, R. Ni, F. Wang, M. Dijkstra, Y. Wang, and Y. Han, Structural signatures of dynamic heterogeneities in monolayers of colloidal ellipsoids, Nat. Commun. 5, 3829 (2014).

[15] J. Roller, A. Laganapan, J.-M. Meijer, M. Fuchs, and A. Zumbusch, Observation of liquid glass in suspensions of ellipsoidal colloids, Proc. Natl. Acad. Sci. 118, e2018072118 (2021).

[16] P. F. Damasceno, M. Engel, and S. C. Glotzer, Predictive selfassembly of polyhedra into complex structures, Science $\mathbf{3 3 7}$, 453 (2012).

[17] R. K. Cersonsky, G. van Anders, P. M. Dodd, and S. C. Glotzer, Relevance of packing to colloidal self-assembly, Proc. Natl. Acad. Sci. 115, 1439 (2018).

[18] Y. Yuan, K. VanderWerf, M. D. Shattuck, and C. S. O'Hern, Jammed packings of 3D superellipsoids with tunable packing fraction, coordination number, and ordering, Soft Matter 15, 9751 (2019).

[19] A. P. Philipse, The random contact equation and its implications for (colloidal) rods in packings, suspensions, and anisotropic powders, Langmuir 12, 1127 (1996).

[20] A. Wouterse, S. R. Williams, and A. P. Philipse, Effect of particle shape on the density and microstructure of random packings, J. Phys.: Condens. Matter 19, 406215 (2007).

[21] K. VanderWerf, W. Jin, M. D. Shattuck, and C. S. O'Hern, Hypostatic jammed packings of frictionless nonspherical particles, Phys. Rev. E 97, 012909 (2018).

[22] C. Brito, H. Ikeda, P. Urbani, M. Wyart, and F. Zamponi, Universality of jamming of nonspherical particles, Proc. Natl. Acad. Sci. 115, 11736 (2018).

[23] C. Song, P. Wang, and H. A. Makse, A phase diagram for jammed matter, Nature (London) 453, 629 (2008).

[24] G. W. Delaney, J. E. Hilton, and P. W. Cleary, Defining random loose packing for nonspherical grains, Phys. Rev. E 83, 051305 (2011).

[25] K. M. Salerno, D. S. Bolintineanu, G. S. Grest, J. B. Lechman, S. J. Plimpton, I. Srivastava, and L. E. Silbert, Effect of shape and friction on the packing and flow of granular materials, Phys. Rev. E 98, 050901(R) (2018).
[26] A. H. Barr, Superquadrics and angle-preserving transformations, IEEE Comput. Grap. Appl. 1, 11 (1981).

[27] G. W. Delaney and P. W. Cleary, The packing properties of superellipsoids, Europhys. Lett. 89, 34002 (2010).

[28] S. Torquato and Y. Jiao, Dense packings of the platonic and archimedean solids, Nature (London) 460, 876 (2009).

[29] Y. Jiao and S. Torquato, Maximally random jammed packings of platonic solids: Hyperuniform long-range correlations and isostaticity, Phys. Rev. E 84, 041309 (2011).

[30] S. A. Galindo-Torres and D. M. Pedroso, Molecular dynamics simulations of complex-shaped particles using Voronoi-based spheropolyhedra, Phys. Rev. E 81, 061303 (2010).

[31] M. Spellings, R. L. Marson, J. A. Anderson, and S. C. Glotzer, GPU accelerated discrete element method (DEM) molecular dynamics for conservative, faceted particle simulations, J. Comput. Phys. 334, 460 (2017).

[32] G. Y. Onoda and E. G. Liniger, Random Loose Packings of Uniform Spheres and the Dilatancy Onset, Phys. Rev. Lett. 64, 2727 (1990).

[33] K. Shundyak, M. van Hecke, and W. van Saarloos, Force mobilization and generalized isostaticity in jammed packings of frictional grains, Phys. Rev. E 75, 010301(R) (2007).

[34] M. Jerkins, M. Schröter, H. L. Swinney, T. J. Senden, M. Saadatfar, and T. Aste, Onset of Mechanical Stability in Random Packings of Frictional Spheres, Phys. Rev. Lett. 101, 018301 (2008).

[35] L. E. Silbert, Jamming of frictional spheres and random loose packing, Soft Matter 6, 2918 (2010)

[36] Y. Yuan et al., Experimental Test on Edwards Volume Ensemble of Tapped Granular Packings, Phys. Rev. Lett. 127, 018002 (2021).

[37] S. Edwards, The equations of stress in a granular material, Physica A 249, 226 (1998).

[38] S. Torquato and Y. Jiao, Effect of dimensionality on the percolation threshold of overlapping nonspherical hyperparticles, Phys. Rev. E 87, 022111 (2013).

[39] S. Henkes, D. A. Quint, Y. Fily, and J. M. Schwarz, Rigid Cluster Decomposition Reveals Criticality in Frictional Jamming, Phys. Rev. Lett. 116, 028301 (2016).

[40] D. Lester and R. Li, The frictional pebble game: An algorithm for rigidity percolation in saturated frictional assemblies, J. Comput. Phys. 369, 225 (2018).

[41] K. Liu, S. Henkes, and J. M. Schwarz, Frictional Rigidity Percolation: A New Universality Class and its Superuniversal Connections Through Minimal Rigidity Proliferation, Phys. Rev. X 9, 021006 (2019).

[42] H. A. Vinutha and S. Sastry, Force networks and jamming in shear-deformed sphere packings, Phys. Rev. E 99, 012123 (2019).

[43] C. E. Zachary, Y. Jiao, and S. Torquato, Hyperuniform LongRange Correlations are a Signature of Disordered Jammed Hard-Particle Packings, Phys. Rev. Lett. 106, 178001 (2011).

[44] Y. Yuan, L. Liu, Y. Zhuang, W. Jin, and S. Li, Coupling effects of particle size and shape on improving the density of disordered polydisperse packings, Phys. Rev. E 98, 042903 (2018).

[45] Y. Yuan, L. Liu, W. Deng, and S. Li, Random-packing properties of spheropolyhedra, Powder Technol. 351, 186 (2019). 
[46] R. Ni, A. P. Gantapara, J. de Graaf, R. van Roij, and M. Dijkstra, Phase diagram of colloidal hard superballs: from cubes via spheres to octahedra, Soft Matter 8, 8826 (2012).

[47] Y. Jiao, F. H. Stillinger, and S. Torquato, Distinctive features arising in maximally random jammed packings of superballs, Phys. Rev. E 81, 041304 (2010).

[48] A. Donev, R. Connelly, F. H. Stillinger, and S. Torquato, Underconstrained jammed packings of nonspherical hard particles: Ellipses and ellipsoids, Phys. Rev. E 75, 051304 (2007).

[49] F. M. Schaller, M. Neudecker, M. Saadatfar, G. W. Delaney, G. E. Schröder-Turk, and M. Schröter, Local Origin of Global Contact Numbers in Frictional Ellipsoid Packings, Phys. Rev. Lett. 114, 158001 (2015).

[50] A. Jaoshvili, A. Esakia, M. Porrati, and P. M. Chaikin, Experiments on the Random Packing of Tetrahedral Dice, Phys. Rev. Lett. 104, 185501 (2010).

[51] M. Neudecker, S. Ulrich, S. Herminghaus, and M. Schröter, Jammed Frictional Tetrahedra are Hyperstatic, Phys. Rev. Lett. 111, 028001 (2013).

[52] K. C. Smith, I. Srivastava, T. S. Fisher, and M. Alam, Variable-cell method for stress-controlled jamming of athermal, frictionless grains, Phys. Rev. E 89, 042203 (2014).

[53] W. Jin, P. Lu, L. Liu, and S. Li, Cluster and constraint analysis in tetrahedron packings, Phys. Rev. E 91, 042203 (2015).

[54] L. Liu, Z. Li, Y. Jiao, and S. Li, Maximally dense random packings of cubes and cuboids via a novel inverse packing method, Soft Matter 13, 748 (2017).

[55] P. Chaudhuri, L. Berthier, and S. Sastry, Jamming Transitions in Amorphous Packings of Frictionless Spheres Occur Over a Continuous Range of Volume Fractions, Phys. Rev. Lett. 104, 165701 (2010).

[56] M. Ozawa, T. Kuroiwa, A. Ikeda, and K. Miyazaki, Jamming Transition and Inherent Structures of Hard Spheres and Discs, Phys. Rev. Lett. 109, 205701 (2012).

[57] C. S. O'Hern, S. A. Langer, A. J. Liu, and S. R. Nagel, Random Packings of Frictionless Particles, Phys. Rev. Lett. 88, 075507 (2002).

[58] M. P. Allen and D. J. Tildesley, Computer Simulation of Liquids (Oxford University Press, Oxford, 2017).

[59] L. E. Silbert, D. Ertaş, G. S. Grest, T. C. Halsey, D. Levine, and S. J. Plimpton, Granular flow down an inclined plane: Bagnold scaling and rheology, Phys. Rev. E 64, 051302 (2001).

[60] D. C. Liu and J. Nocedal, On the limited memory BFGS method for large scale optimization, Math. Program. 45, 503 (1989).

[61] K. Wang, C. Song, P. Wang, and H. A. Makse, Edwards thermodynamics of the jamming transition for frictionless packings: ergodicity test and role of angoricity and compactivity, Phys. Rev. E 86, 011305 (2012).

[62] M. Mailman, C. F. Schreck, C. S. O'Hern, and B. Chakraborty, Jamming in Systems Composed of Frictionless Ellipse-Shaped Particles, Phys. Rev. Lett. 102, 255501 (2009).

[63] Z. Zeravcic, N. Xu, A. Liu, S. Nagel, and W. van Saarloos, Excitations of ellipsoid packings near jamming, Europhys. Lett. 87, 26001 (2009).

[64] K. C. Smith, T. S. Fisher, and M. Alam, Isostaticity of constraints in amorphous jammed systems of soft frictionless platonic solids, Phys. Rev. E 84, 030301(R) (2011).

[65] J. Zhao, S. Li, R. Zou, and A. Yu, Dense random packings of spherocylinders, Soft Matter 8, 1003 (2012).

[66] N. Gravish, S. V. Franklin, D. L. Hu, and D. I. Goldman, Entangled Granular Media, Phys. Rev. Lett. 108, 208001 (2012).

[67] K. Shiraishi, H. Mizuno, and A. Ikeda, Mechanical and vibrational properties of three-dimensional dimer packings near the jamming transition, J. Phys. Soc. Jpn. 89, 074603 (2020).

[68] T. M. Truskett, S. Torquato, and P. G. Debenedetti, Towards a quantification of disorder in materials: Distinguishing equilibrium and glassy sphere packings, Phys. Rev. E 62, 993 (2000).

[69] P. K. Morse and E. I. Corwin, Echoes of the Glass Transition in Athermal Soft Spheres, Phys. Rev. Lett. 119, 118003 (2017).

[70] A. Ikeda, L. Berthier, and G. Parisi, Large-scale structure of randomly jammed spheres, Phys. Rev. E 95, 052125 (2017).

[71] L. E. Silbert and M. Silbert, Long-wavelength structural anomalies in jammed systems, Phys. Rev. E 80, 041304 (2009).

[72] D. Hexner and D. Levine, Hyperuniformity of Critical Absorbing States, Phys. Rev. Lett. 114, 110602 (2015).

[73] D. Hexner, P. M. Chaikin, and D. Levine, Enhanced hyperuniformity from random reorganization, Proc. Natl. Acad. Sci. 114, 4294 (2017).

[74] C. Ness and M. E. Cates, Absorbing-State Transitions in Granular Materials Close to Jamming, Phys. Rev. Lett. 124, 088004 (2020).

[75] M. A. Klatt, J. Lovrić, D. Chen, S. C. Kapfer, F. M. Schaller, P. W. Schönhöfer, B. S. Gardiner, A.-S. Smith, G. E. SchröderTurk, and S. Torquato, Universal hidden order in amorphous cellular geometries, Nat. Commun. 10, 811 (2019).

[76] J. R. Royer and P. M. Chaikin, Precisely cyclic sand: Selforganization of periodically sheared frictional grains, Proc. Natl. Acad. Sci. 112, 49 (2015).

[77] B. Kou, Y. Cao, J. Li, C. Xia, Z. Li, H. Dong, A. Zhang, J. Zhang, W. Kob, and Y. Wang, Granular materials flow like complex fluids, Nature (London) 551, 360 (2017). 